\title{
Kikuchi's Disease: A Case Report
}

\author{
MSU Rahman ${ }^{1}$, G Muktadir², SAM Ahmed ${ }^{3}$
}

\begin{abstract}
:
Kikuchi's disease is also known as histiocytic necrotizing lymphadenitis. Kikuchi-Fujimoto disease (KFD) or histiocytic necrotizing lymphadenitis, is a rare benign, self-limiting cervical lymphadenitis of unknown etiology. It predominantly affects young women and can closely mimic infective and immunological disorders. Recognition of this condition is crucial, specially because it can easily be mistaken for tuberculosis, lymphoma, or even adenocarcinoma. We report an 18 years old girl who presented with fever and cervical lymphadenopathy. She had multiple enlarged cervical lymphadenopathy. Examination of other systems was normal. Laboratory investigations were also normal. Fine needle aspiration cytology of the cervical node was not done. The patient took a course of oral antibiotics. Since the patient did not respond, lymph node biopsy was done and the histological features suggested the diagnosis of Kikuchi's disease. The Patient was treated symptomatically and complete remission occurred in few weeks. Although the incidence of Kikuchi-Fujimoto disease is rare, clinicians should be aware of this condition as early recognition of the disease will minimize potentially harmful and unnecessary evaluations and treatments.
\end{abstract}

Key words: Kikuchi-Fujimoto disease, Histiocytic Necrotizing lymphadenitis, Systemic Lupus Erythematosus.

\section{Introduction :}

Kikuchi-Fujimoto disease (KFD) or histiocytic necrotizing lymphadenitis is an uncommon, idiopathic, generally self-limited cause of lymphadenitis ${ }^{1,2}$. Kikuchi first described the disease in 1972 in Japan. Fujimoto and colleagues independently described Kikuchi's disease in the same year. Kikuchi-Fujimoto disease is mainly seen in Japan. Isolated cases are reported in America, Europe and Asia. It is mainly a disease of young adults (mean age, 20-30 years), with a slight bias towards female ${ }^{3}$. Compared to male, ratio is of $1: 4^{4}$. The cause of this disease is not known although infection and autoimmune etiologies have been proposed. Course of the disease is generally benign and self-limiting. Lymphadenopathy most often resolves over several weeks to 6 months. Recurrence rate is about $3 \%{ }^{4}$. Mortality is extremely rare and usually due to hepatic, respiratory or cardiac failure. Awareness of this disorder will help to prevent misdiagnosis and inappropriate treatment.

1. Dr. Md. Shafiq-Ur Rahman, MBBS, DLO, Assistant Professor, Department of Otorhinolaryngology, Faridpur Medical College, Faridpur.

2. Dr. Golam Muktadir, MBBS, DLO, MS (ENT), Associate Professor and Head, Department of Otorhinolaryngology, Faridpur Medical College, Faridpur.

3. Dr. SK. Abdul Momen Ahmed, MBBS, MPH (Community Medicine), MD (Pathology), Lecturer, Department of Pathology, Faridpur Medical College, Faridpur.

Address of correspondence :

Dr. Md. Shafiq-Ur Rahman, MBBS, DLO, Assistant Professor, Department of Otorhinolaryngology, Faridpur Medical College, Faridpur. Mobile No: +8801715073840 ,

Email: shafiqur.rahman.52@facebook.com

\section{Case Report :}

An 18 year old girl Mst. Jannati of Saltha, Faridpur district presented to us with multiple neck swellings and fever of 2 months duration. There was no weight loss. There was no previous history of tuberculosis or contact with tuberculosis. She did not have history of any drug intake or atopy. She did not have any other significant medical problems.

Clinical Examinations revealed bilateral large, mobile and tender-cervical lymphadenopathy, larger node being the right posterior triangle of neck which was measured about $2.5 \times 4 \mathrm{~cm}$, other enlarged nodes were right posterior cervical $(2 \times 2.2 \mathrm{~cm})$, left posterior auricular, left submandibular and right submandibular region. Lymph nodes were not palpable in other parts of the body. The blood pressure was 110/70 $\mathrm{mm}$ of $\mathrm{Hg}$ and the pulse rate was $80 / \mathrm{min}$. Her cardiovascular, respiratory and neurological examinations were normal. The abdomen was soft with normal bowel sounds. Ear, nose and throat examinations were normal. Routine haematological parameters like hemoglobin, complete blood count, peripheral smear were within normal limits. ESR was $20 \mathrm{~mm}$ in 1st hour. Blood glucose, urea, creatinine, sodium, potassium and bicarbonate levels were normal. Renal and liver function tests were normal. Blood and urine cultures were negative. Montoux test showed induration of $4 \mathrm{~mm}$. Ultrasound of abdomen and Chest radiograph was normal. Antinuclear antibody (ANA) and anti-ds DNA antibody were negative. Staining for AFB (acid-fast bacilli) was 
also negative. Fine needle aspiration cytology (FNAC) of lymph node was not done. The potient was started an oral antibiotic (cap, cefixime-200mg 12 hourly for 7 days). Since the patient oontinued to have fever and persistent lymphadenopathy despite one week of antibiotic, lymph node biopsy was done and the histological features suggested the diagnosis of Kikuchi-Fujimoto disease (Figure 1). The potient was treated symptomatically with non-steroidal antiinflammatory drugs, the lymph nodes regressed in one month.

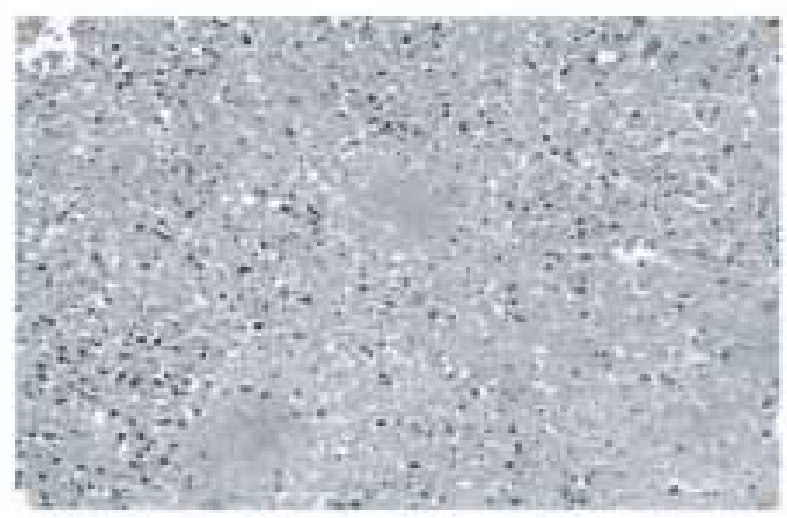

Figure II Lymph node blopsy sectlon showing patchy areas of necrosis, proliferation of pale histicestes, increase number of apoptotic cells; cellular detris and nuclear dust (kuryorrhedis)(H\&E, xuoo)

\section{Discussion :}

Kikuchi's disease most often presents with cervical lymphadenopathy which may be tender or non-tender and ean be accompanied by fever, upper respiratory tract symptoms. Less common symptoms include arthralgia, skin rashes, weakness and night sweats. Weight loss, diarthoea, anorexia, chills, nausea, vomiting, chest and abdominal pain have also been reported. Some patients may also bave hepatosplenomeguly $y^{3-7}$. Involvement of skin and bone marrow has also been reported in some cases ${ }^{89}$. The exact aetiology of Kikuchi's disease is not known. Viral agents such as Epstein barr virus (EBV), Human immunodeficiency virus (HIV), Herpes simplex virus, dengue vinus, Human T lymphotrophic virus I (HTLV1) and Parvovirus B19 have been suggested as possible aetiological agents, but none have been confirmed so far ${ }^{4}$. Toxoplasma and sther becterial agents like Yersinia enterocolitica, Bartonella, Bracella have also been implemented". An autoimmune mechanism has also been proposed because KFD is seen in conjunction with systemic lupus erythematosus (SLE). There are several reports suggesting an association between Kikuchi's disease and systemic lupus erythematosus (SLE). However no convinicing evidence is available to confirm such association. The pathogenesis of Kikuchi's disease is still not fully clear. It is supposed that the primary event may be the activation of $\mathrm{T}$ lymphocytes and histiocytes. Proliferating T cells enter the cycle of apoptosis, which may form the areas of necrosis in lymph nodes and then the cellular debris is removed by histiocytes ${ }^{10}$.

Routine laboratory investigutjons usually does not aid in the diagnosis of KFD except for erythrocyte sedimentation rate (ESR) and C-reactive protein (CRP) may be elevated in some cases and many patients have a low white blood count. Moreover, $25 \%$ to $31 \%$ of patients have atypioal peripheral blood lymphocytes" Fine-necdle asparation eytology (FNAC) only has a limited role in establishing the diagnosis of Kikuchi's disease. Diagnosis is based on histopathological findings of a lymph node biopoy.

Clinically Kikuchi's disease may mimic systemic lupus erythematosus (SLE) or Lymphoma (specially T-Cell non-Hodgkin's Lymphoma) as both this diseases can present with lymphadenopathy and fever and the skin lesions of Kikuchi's discase putients can resemble those scen in SLE. Careful histopathologic examination will thus help us distinguish KFD from other diseases. Histological feature which helps in the differentiation of KFD from the lymphadenopathy of systemic lupus erythematosus is almost total absence of plasma cells in the involved nodal tissue. Moreover appropriate serologic tests should be done to exclude systemic lupus erythematosus ${ }^{12}$. Features that distinguish KFD from malignant lymphoma inclade incomplete architectural effacement with patent sinuses, presence of numerous reactive histocytes, relatively low mitotic rates, absence of Reed- Sterberg cell ${ }^{13}$.

The CT appearance of Kikuchi's disease may be variable, mimioking various nodal diseases, such as tuberculosis, metastasis and lymphoma. The necrotic areu of the lymph nodes had a lower signal than the non-necrotic portion on $\mathrm{T}_{2}$-weighted $\mathrm{MR}$ images, which is different from the usual bright signal, intensity of nodal necrosis seen in otber nodal diseuses.

No specifie treutment is available for Kikuchi's disease. Treatment is largely supportive. Non-steroidal antiinflammatory drugs (NSAIDs) for tender lymph nodes and fever. The ase of corticosteroids bas been recommended in severe form of disease ${ }^{14}$. Intruvenous immunoglobulin has also been tried with some success ${ }^{15}$. The disease usually runs a benign course and the oundition is self-limiting, usually resolves in several weeks to months. The disease bus a recurrenoes rate of $3 \%$ to $4 \%{ }^{*}$. 


\section{Conclusion :}

Although the incidence of Kikuchi's disease is rare, this disorder must be considered among the differential diagnosis when a young female patient presents with fever and cervical lymphadenopathy. Clinically Kikuchi's disease may mimic lymphoma or systemic lupus erythematosus (SLE). Therefore a careful histopathological examination is necessary in arriving at the diagnosis. Early recognition of the disease is very necessary for harmful and unnecessary evaluations and treatments.

\section{References :}

1. Kikuchi M. Lyphadenitis showing focal reticular cell hyperplasia with nuclear debris and phagocytes: a clinicopathological study. Acta Hematol Jpn. 1972: 35:379-80.

2. Fujimoto Y, Kozima Y, Yamaguchi K. Cervical subacute necrotizing lymphadenities; a new clinicophathologic entity. Naika 1972: 20:920-27.

3. Kuo T. Kikuchi's disease histocytic necrotizing lymphadenitis: A clinicopathologic study of 79 cases with an analysis of histocytologic subtypes, immunohistology and DNA Ploidy. Am j. burg Pathol. 1995: 19:798-809.

4. Sudhakar MK, Sathyamurthy P, Indhumathi E, Amarabalan R, Bavya V. Kikuchi's Disease: A case report from south India. IJCRL 2011: 2 (2):15-18.

5. Dorfman RF, Berry GJ. Kikuci's histiocytic necrotizing lymphadenitis: an analysis of 108 cases with emphasis on differential diagnosis. Sermin Dign Pathol. 1988: 5:329-45.

6. Chamuk G, Brynes R, Nath WB. Kikuchi-Fujimoto diseases mimicking malignant lymphoma. Am J Surg Pathol. 1990:14:514-23.

7. Rudniki C, Kessler E, Zarfati M, Turani H, Bar-Ziv Y, Zahavi I. Kikuchi's necrotizing Lymphadenitis: a cause of fever of unknown origin and splenomegaly. Acta Hematol. 1988: 7 (9):99-102.

8. Sumioyoshi Y, Kikuchi M, Oshima K, et al. A case of histiocytic necrotizing lymphadenities with bone marrow and skin involvement. Virchows arch. 1992: 420:275-79.

9. Sousa AA, Soares JM, de sa Santos MH, Martins MP, Salles JM. Kikuchi-Fujimoto disease; three cases reports. Sao Paulo med. J. 2010; 128 (4):798-809.

10. Hrycek A, Cieslik P, Witold S, Jacek P. Kikuchi's Fujimoto disease; a case report. Rheumatil int. 2005; 26:179-81.

11. Mosharrof HAK, Datta PG, Amin AS, Uddin MJ. KikuchiFujimoto Diseases presenting with fever, lymphadenopathy and dysphagia. J Pak. Med Assoc. 2008: 58:647-49.

12. Louis N, Hanely M, Davidson UM. Kikuchi-Fujimoto disease: a report of two cases and an overview. The Journal of Laryngology otology 1994; 108:1001-1004.doi: 10. 1017/ soo221510012.8749.
13. Bosch X, Guilbert. Kikuchi-Fujimoto diseases. Prphanet J Rare Dis. 2006; 1:18. doi: 10. 1186/ 1750-1172-1-18.

14. Jang YJ, Park KH, Seok HJ. Management of Kikuchi's disease using glucocorticoid. J Laryngol. 2000; 114:709-11.

15. Noursadeghi M, Aqel N, Gibson P, Pasvol G. Successful treatment of severe Kikuchi's disease with intravenous immunoglobulin. Rheumatology 2005; 45:35-37. 